\title{
Simultaneous Distribution Network Reconfiguration and Optimal Deployment of Distributed Generation
}

\author{
A. B. Kunya ${ }^{1, *}$, G. S. Shehu' ${ }^{1}$, U. M. Hassan ${ }^{1}$, A. U. Lawan ${ }^{2}$ (D) \\ ${ }_{1}^{1}$ Department of Electrical Engineering, Ahmadu Bello University, Zaria-Nigeria \\ ${ }^{2}$ Department of Electrical Engineering, University of Nottingham Malaysia Campus, Malaysia
}

\begin{abstract}
A reliable, eco- and nature-friendly operation has been the major concern of modern power system (PS). To enhance the PS reliability and reduce the adverse environmental effect of conventional thermal generation facilities, renewable energy based distributed generation (RDG) are being enormously integrated to low and medium voltage distribution networks (DN). However, if the RDGs are poorly designed or placed, the system will be vulnerable to instability issues which ultimately jeopardizes the quality. Part of the measures used to avert such is optimizing the RDGs capacity and point of integration to the DNs. These DNs are generally operated on a radial configuration, though they can be reconfigured to other topologies to achieve certain objectives. Both RDG placement/sizing and DN reconfiguration are multi-objective and combinatorial optimization problems. In this study, a hybrid of Particle Swarm Optimization (PSO) and real-coded Genetic Algorithm (GA) techniques is employed for DN reconfiguration and optimal allocation of three RDG units in primary DNs simultaneously. The objectives of the proposed technique are to minimize active power loss (PL), improve voltage profile (VP) and enhance feeder load balancing (LB). For validation purpose, the proposed technique is implemented on 16-bus (3-feeder), single feeder 33-bus and 69-bus IEEE test DNs. It is implemented in MATLAB interfaced with MATPOWER simulation packages. It is observed the PL, VD and LB are found to be reduced by $32.84 \%, 12.33 \%$ and $24.03 \%$ of their respective inherent values in the biggest system when the system is reconfigured only. With the optimized RDGs placed in the reconfigured systems, a further reductions of $46.27 \%, 25.92 \%$ and $36.65 \%$ are observed respectively.
\end{abstract}

Key words: Feeder Load Balancing, Distribution Network Reconfiguration, Genetic Algorithm, Loss Reduction, Particle Swarm Optimization, Voltage Deviation.

\section{INTRODUCTION}

Traditional power systems (PSs) are designed to accommodate power flow in one direction. However, owing to the some economic and environmental concerns like escalation in the cost of fuel and greenhouse gas emissions, renewable energy based distributed generations (RDGs) are adopted as one of the promising alternative to fossil fuels [1]. Even though integration of RDGs to distribution networks (DNs) constitutes bidirectional power flow, it can facilitate implementation of competitive energy policies, diversify the available energy sources, deferment of PS facilities upgrades and improve system efficiency through reduction of energy losses [2]. Despite the numerous economic and technical benefits derived from RDG, if they are poorly designed and/or placed, the system will be vulnerable to instability issues which ultimately jeopardizes the quality like increases losses, injects harmonic currents and deteriorates voltage profile (VP). Conversely, optimizing the RDG size and location in DN can improve network performance in terms of VP, reduced cost of energy. In addition, these improvements in the PS quality and many more can be equally achieved when the topology of the DN is optimally restructured or reconfigured.

Generally, DNs are built as weakly meshed networks but operated in a radial configuration, though they can be reconfigured to other topologies to achieve certain objectives $[3,4,5]$. DN reconfiguration (DNR) is the technique of modifying the topological structure of $\mathrm{DN}$ by varying the open/close states of the network sectionalizing and tie switches [6]. Sectionalizing switches are normally closed switches placed within each feeder of the DN while tie switches are normally open switches connecting part of one feeder to another [7]. DNR is an effective way of improving the performance of power DNs such as reducing power losses $[8,9]$, voltage profile (VP) improvement $[10$, $11]$, feeder load balancing (LB) $[9,12]$, increasing RDG penetration $[13,14]$, mitigating operational constraint violations [12], service restoration $[15,16]$, improving system security [17] and relieving primary feeders for the purpose of repairs on that feeder [18]. The DN reconfiguration changes the power flow direction which subsequently alters the line currents, bus voltages, level of symmetry and level of harmonic distortion on the bus voltages $[19,20]$.

DNR is carried out either offline better known static DNR or online called dynamic DNR. However, due to intermittent nature of load demand and RDGs power output, static DNR is seldom applied hence necessitating the dynamic reconfiguration. In dynamic DNR, the $\mathrm{DN}$ operators can restructure the topology of DNs in real time by switching the remotely controlled switches on or off [14].

$\begin{array}{ll}\text { Received } & \text { : May 25, 2019 } \\ \text { Revised } & : \text { September 18, 2019 } \\ \text { Accepted } & : \text { September 25, 2019 }\end{array}$


Furthermore, both RDG placement/sizing and DNR are highly non-linear, multi-objective, constrained and combinatorial optimization problems. The complexity increases in manifold when the two problems are solved simultaneously. There are numerous research works carried out on these techniques separately. However, the determining the optimal DNR and RDG placement/sizing simultaneously is still rarely available in the literature. The research work presented the solution of DNR for loss reduction was pioneered by Merlin and Back [21]. Spanning tree approach was proposed where all the switches in the $\mathrm{DN}$ were closed and then opened successively to eliminate the loops. Subsequently, many other algorithms were developed to solve this problem. For instance, PL minimization and VD improvement using DNR is presented in [22]. Likewise, in [23], DNR is applied to DNs to maximize the DN's hosting capacity for plug-in electric vehicles and minimize costs. In the authors' previous work [10], particle swarm optimization (PSO) is applied to solve optimal DNR for loss reduction and voltage profile improvement. Similarly, other heuristic and meta-heuristic optimization techniques were applied for DNR studies like hybrid evolutionary algorithm [24], non-dominated sorting genetic algorithm-II [25], plant growth simulation algorithm [26], ant colony algorithm [27] and multi objective honey bee mating optimization [28]. In [29], a nature-inspired algorithm is applied for the solution of complex DNR with multiple objectives. The hybrid artificial immune systems-ant colony optimization approach is selected in the work due to its ability to handle the multi-objective formulations and to provide restoration solution during contingencies. Graph theory is applied both in [30] and [31] with a view of improving the computational performance of the DNR technique, while the artificial immune system and multi-objective discrete PSO are used to solve the problem of multi-objective DNR, correspondingly.

On the other hand, there are number of works done on the optimal allocation of RDG. For instance, in [32] optimal RDG placement in cyber-physical PS is presented. Ant-lion optimization technique is applied in [33] to determine the optimal size and location of RDG with the aim of reducing the purchased energy cost from upstream, reliability improvement, losses and voltage deviation reduction. Optimal placement and sizing of multiple RDG units is presented using by invasive weed optimization algorithm in [34]. In spite of the improvements achieved in the respective objectives of the aforementioned researches, none solved the DNR and RDG allocation simultaneously. Recently, attempts were made to solve the two problems simultaneously like in $[35,36]$. The objective of these techniques is minimizing the PL and improve VP. However, due to high combinations of the solution space, search space reduction technique is applied to phase out some solutions.

In this proposed study, a hybrid PSO and real-coded Genetic Algorithm (HPSOGA) technique is employed for optimal DNR and multiple RDG units placement and sizing in primary DNs simultaneously. The two optimization techniques are hybridized to reap the advantages of both techniques; faster convergence of PSO and the ability of GA to avoid trapping in local minima. The objectives of the proposed technique are to simultaneously minimize PL, improve VP and enhance feeder load balancing (LB). It is carried out subject to some technical constraints like limits of bus voltage magnitude, maximum permissible line current carrying capacity and radial network structure (RNS). The search space of the proposed work is the set of DN branches, DG sizes and potential locations.

The significant contribution of this study are itemized as follows:-

- Proposes a hybrid of PSO and GA for the solution for DNR and RDG allocation simultaneously.

- Formulates the technique with the capability of solving the both RDG deployment and DNR on DNs with single and multiple feeder (s).

- Determines the optimal solutions devoid of search space reduction.

The rest of the paper is prepared the Formulation of the HPSOGA presented in Section II. The problem formulation is comprehensively discussed in Section III, while the test system are described in Section IV. Section V presents the tests, results, and discussion and the paper is concluded in Section VI.

\section{HYBRID PSO WITH GA}

Particle swarm optimization (PSO) is a population based technique that mimic the behavior of the birds or fish (called particles represented by dimensional vectors) in a swarm. In the search space, each particle changes position at certain rate. The particle familiarizes with this rate of change in position while swapping information over with its neighboring particles. Iteratively, each particle memorizes its best position and the overall best particle positions. The best particle position is saved as a best local position, which was assigned to a neighborhood particles, while the overall best particle position is saved as a best global position, which was assigned to all particles in the swarm. The major downside of PSO is that the swarm may converge earlier than expected. Due to the fast rate of information flow between particles, resulting in the creation of similar particles with a loss in diversity that increases the possibility of being trapped in local optima. The underlying principle behind PSO formulation can be found in $[37,25]$.

On the other hand, Genetic algorithm (GA) essentially a flexible heuristic search algorithm developed based on the evolutionary concept of natural selection and genetics. It applies a crossover operator by mating the parents (individuals) and a mutation operator that stochastically updates the individual contents to diversify generation of new offspring. The replacement (survival selection) is generational, that is, the parents are replaced systematically by the offspring. As a global search optimization technique, GA often skips local optimal solutions by reproducing solutions stochastically. The underlying principle behind GA formulation can be found in $[37,25]$.

The two algorithms are combined to form a hybrid algorithm. The fundamental concept behind the proposed HPSOGA algorithm is summarised as;

Step 1: Initialize the values of the particle dimension $P$, constants of rate of velocity change; $c_{1}$ and $c_{2}$, 
crossover probability $P_{\mathrm{c}}$, mutation probability $P_{\mathrm{m}}$,

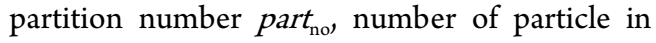
each partition $m$, number of solutions in each partition $g$ and the maximum number of iterations max $_{\text {itr. }}$.

Step 2: Set the initial counting index, $t=0$;

Step 3: for $1 \leq i \leq \mathrm{p}$, do

Step 4: Compute an initial population $\overrightarrow{X_{l}(t)}$ arbitrarily.

Step 5: Compute the fitness function of each set of variable, $f\left(\overrightarrow{X_{l}}\right)$ :

Step 6: end for

Step 7: go to step 3.

Step 8: Apply the standard particle swarm PSO algorithm on the whole population $\overrightarrow{X(t)}$

Step 9: Apply the GA selection operator on the entity of the population, $\overrightarrow{X(t)}$

Step 10: Divide the population $\overrightarrow{X(t)}$ to part $_{\mathrm{no}}$ subpartitions, where each sub-partition $\overrightarrow{X^{\prime}(t)}$ size is $m x \eta$;

Step 11: for $1 \leq i \leq$ part $_{\mathrm{no}}$, do

Step 12: Apply the arithmetic crossover on each sub-partition;

Step 13: end for

Step 14: Apply the GA $\overrightarrow{X(t)}$ mutation operator on the whole population

Step 15: Update the solutions in the population

Step 16: Increase the iteration counter; Set $t=t+1$

Step 17: until $t>\max _{i t r}$ \{Termination criteria are satisfied\};

Step 18: Print out the best solution.

\section{PROBLEM FORMULATION}

In this study, the HPSOGA is employed for determining the optimal DN reconfiguration and as well as the size and location of multiple RDG units in primary DNs simultaneously, while satisfying different technical constraints. While formulating the proposed technique, there are some assumptions made, as summarised below:

- Only single RDG unit can be allocated to each potential candidate bus. Thus, no bus in the DN can take more than one RDG unit.

- The DNR as well as the RDG placement is assumed to be offline, as such rated load demands are used in the model as an alternative of time-varying demands.

\section{A. Objective Function}

The objectives of the proposed technique are to minimize the active PL, improve VP and enhance feeder LB. Thus, the objective function has three terms as formulated in (1).

Where

$$
\min F
$$

$$
F=\psi f_{1}+\beta f_{2}+\xi f_{3}
$$

The first part of the objective function depicts the power loss component obtained by normalizing the PL with its original value as expressed in (2),

$$
f_{1}=\frac{P_{\text {loss }}}{P_{\text {loss }}^{\text {initial }}}
$$

where $P_{\text {loss }}$ is the power loss experienced in the optimized $\mathrm{DN}$, while $P_{\text {initial loss }}$ is its inherent power loss calculated as;

$$
P_{\text {loss }}^{\text {initial }}=\sum_{i=1}^{n_{l}} R_{i}\left\{\frac{P_{i}^{2}+Q_{i}^{2}}{V_{i}^{2}}\right\} \text { for } i=1,2, \ldots, n_{l}
$$

The second part of the objective function, $f_{2}$ is reflects the component of the total voltage deviation, which when minimized, the VP improves. It is similarly obtained by normalizing its minimized value, $V_{d e v}$ with its initial value, $V_{\text {initial der }}$

$$
f_{2}=\frac{V_{\text {dev }}}{V_{\text {dev }}^{\text {initial }}}
$$

The total inherent voltage deviation is computed as;

$$
\Delta V_{\text {total }}^{\text {initial }}=\sum_{j=1}^{n_{b}}\left|V_{\text {ref }, j}-V_{j}\right| \quad \text { for } j=1,2, \ldots n_{b}
$$

$V_{\text {ref }, j}$ is the nominal voltage magnitude of load bus $j$. The third term of the objective function denotes for the LB component of the lines in the feeders, which is shown in (6) [2].

$$
L B=\sum_{j=1}^{n_{f}} \sum_{i=1}^{n_{l}}\left(\frac{n_{f} I_{i}}{\sum_{i=1}^{n_{f}} I_{i}}\right)^{2}
$$

Where $n_{l}$ and $n_{f}$ are the total number of lines (branches) and feeders in the DN respectively. $\psi, \beta$ and $\xi$ are the weighing coefficients of the power loss, voltage deviation and LB components of the objective function respectively. Since there are different objectives to be satisfied simultaneously, after normalizing all the terms of the objectives, the weighing coefficients are chosen thoughtfully to avoid convergence problem.

\section{B. Constraints}

The proposed simultaneous $\mathrm{DN}$ reconfiguration and $\mathrm{RDG}$ units allocation in primary $\mathrm{DNs}$ is solved by considering the following constraints:

1. Voltage limit: the bus voltage magnitudes are constrained within lower and upper limits as in (7),

$$
V_{i}^{\min } \leq V_{i} \leq V_{i}^{\max }, \quad i=1,2, \ldots, n_{b}
$$

2. Maximum permissible line current carrying capacity: to avoid exceeding the current capacity of the branches, the current in $k$ th branch, $I_{k}$ is limited to a maximum permissible current, $I_{\min k}$ as in (8),

$$
I_{k} \leq I_{k}^{\min }, \quad k=1,2, \ldots, n_{l}
$$

Where

$$
\begin{gathered}
I_{k}^{2}=\alpha_{k}\left[A_{i j} V_{i}^{2}+B_{i j} V_{i}^{2}-2 V_{i} V_{j}\left(C_{i j} \cos \beta_{i j}-D_{i j} \sin \beta_{i j}\right)\right] \\
A_{i j}=g_{i j}^{2}+\left(b_{i j}+\frac{b_{s h i j}}{2}\right)^{2} \\
B_{i j}=g_{i j}^{2}+b_{i j}^{2} \\
C_{i j}=g_{i j}^{2}+b_{i j}\left(b_{i j}+\frac{b_{s h i j}}{2}\right)^{2} \\
D_{i j}=g_{i j}^{2} \frac{b_{s h i j}}{2}
\end{gathered}
$$


3. Radial network structure (RNS)

To preserve the radial topology of the $\mathrm{DN}$, no single loop is allowed in the network and at the same no islanded bus. This is represented as:

$$
n_{l}^{\text {active }}=n_{l}-1
$$

Where $n_{\text {active I }}$ is the total number of tie branches, expressed as

$$
\sum_{i=1}^{n_{b}} A(i, j) \geq 1 ; \quad j=1,2, \ldots, n_{b}-1
$$

where $A$ is the reduced node incidence matrix of the $\mathrm{DN}$.

\section{Power Flow Solutions}

Load flow analysis is needed to get the initial voltages, powers and the rest of the parameters (2) - (11). DNs have radial structure, therefore its $R / X$ ratio is quite high, as such have their admittance matrices are tri-diagonal matrices. Therefore, DNs are ill-conditioned, hence conventional load flow techniques are ineffective in analysing such networks. In this study, backward-forward sweeping technique is used.

\section{TEST SYSTEM DESCRIPTION}

To verify the efficacy of the proposed technique and its application to DNs, it is implemented on 16-bus (3-feeder), single feeder 33-bus and 69-bus IEEE test DNs. The 16-bus DN has 3 feeders and radial topology with a total real and reactive loads of $28.125 \mathrm{MW}$ and $13.53 \mathrm{MVAr}$ on $12.65 \mathrm{kV}$ substation voltage respectively.

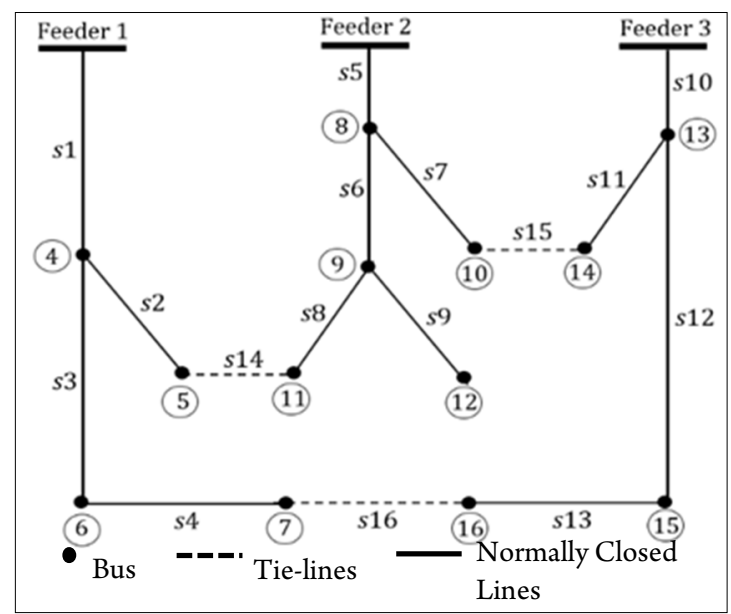

Fig. 1. Single line diagram of IEEE 16-bus DN

The branches linking two nodes has a sectionalizing switch for the nodes connected to the same feeder and a tie switch for the nodes linked to two distinct feeders as shown in Fig. 1. So, the network has a total of 3 tie switches and 14 sectionalizing switches are used in this system [38].

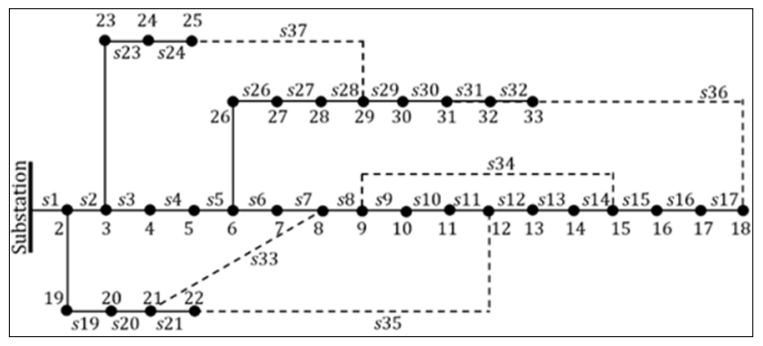

Fig. 2. Single line diagram of IEEE 33-bus DN
The second DN used in this work is 33-bus, single feeder radial DN with a total active and reactive load demand of $3.72 \mathrm{MW}$ and $2.30 \mathrm{MVAr}$ on $12.66 \mathrm{kV}$ respectively [6]. It has 37 branches, 32 sectionalizing switches and 5 tie switches as shown in Fig. 2.

A relatively bigger system with 69 buses, 73 branches and 5 tie switches, is also used. The DN has a total load demand of 3.80MW and 2.70MVAr. The single line diagram of the system is depicted in Fig. 3 [1].

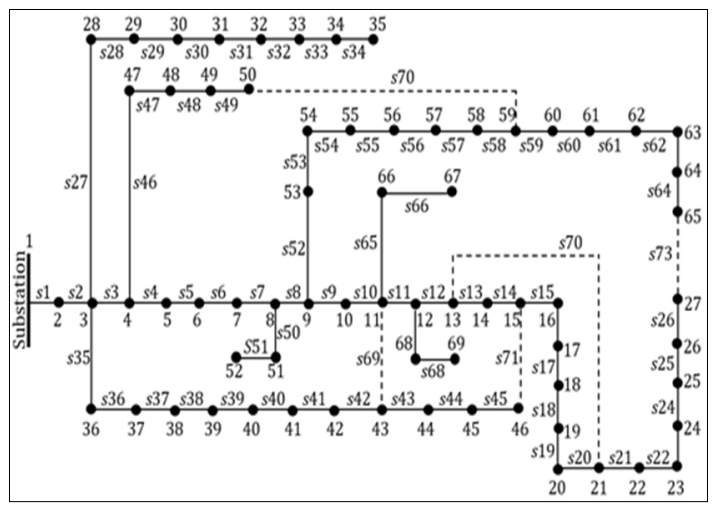

Fig. 3. Single line diagram of IEEE 69-bus DN

\section{SIMULATION RESULTS AND DISCUSSIONS}

The efficiency of the technique is ascertained on the abovementioned test systems. The improvement obtained in the specific objectives are expressed as the percentage of their respective original values.

\section{- 16-bus, 3-feeder DN}

The developed technique determined the optimal DNR by varying changing status of the switches and searching candidate buses for RDG placement and sizing to minimize the objective function while abiding by the rest of the constraints. The optimal DNR is obtained by closing $s 14$ and $s 15$ and opening $s 7$ and $s 8$ while maintaining $s 16$ open as shown in Fig 4. While the optimal RDG location are buses 7, 10 and 12 with respective sizes of $0.31 \mathrm{MW}, 0.23 \mathrm{MW}$ and $0.18 \mathrm{MW}$

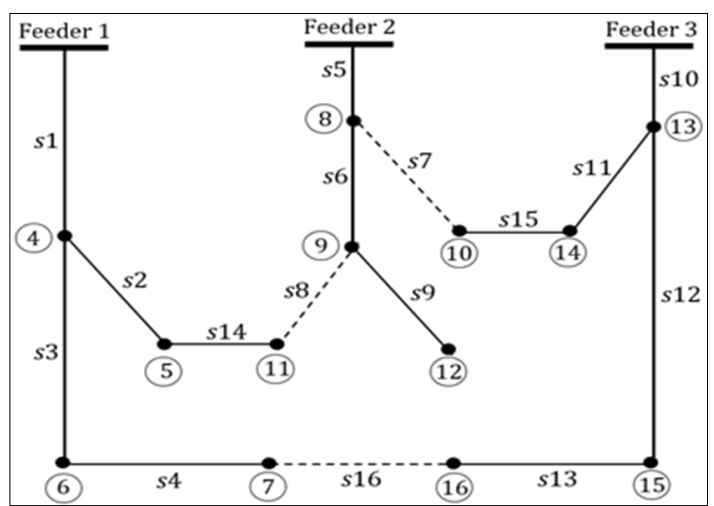

Fig. 4. Optimized single line diagram of 16-bus DN

With reconfiguration alone, the power loss is found to be reduced by $8.89 \%$ of the total loss established to be $514.0293 \mathrm{~kW}$. The voltage deviation reduced by $12.33 \%$ of its inherent value of $0.2140 \mathrm{pu}$. While the LB index is reduced from 56.42 to 49.66 . However, when the DNR and RDG placement are carried out simultaneously, further improvement is observed in the VP, loss reduction and LB as summarized in Table I. 
Table 116 Buss Test System Results

\begin{tabular}{lccc}
\multicolumn{1}{c}{ Parameters } & Initial & Reconfigured & Reconfigured with RDG \\
\hline Tie switches & $14,15,16$ & $7,8,16$ & - \\
RDG location (Size) & - & - & bus7 (0.31MW), bus10 (0.23MW), bus12(0.18MW) \\
Power loss & $514.0293 \mathrm{~kW}$ & $468.3304 \mathrm{~kW}$ & $396.83 \mathrm{~kW}$ \\
$\%$ reduction & - & $8.89 \%$ & $22.8 \%$ \\
Voltage dev. & $0.2140 \mathrm{pu}$ & $0.1876 \mathrm{pu}$ & $0.158 \mathrm{pu}$ \\
\% Improvement & - & $12.33 \%$ & $26.41 \%$ \\
LB index & 56.42 & 48.66 & 40.54 \\
\% Improvement & - & $13.75 \%$ & $28.15 \%$ \\
Min. Voltage & $0.9662 \mathrm{pu}$ & $0.9720 \mathrm{pu}$ & $0.9896 \mathrm{pu}$ \\
\end{tabular}

Table 2 33-Bus Test System Results

\begin{tabular}{lccc}
\hline \multicolumn{1}{c}{ Parameters } & Initial & Reconfigured & Reconfigured with RDG \\
\hline Tie switches & $33,34,35,36,37$ & $7,11,14,28,32$ & - \\
RDG location (Size) & - & - & Bus14 (0.22MW), bus28 (0.30MW), bus33 (0.24MW) \\
Power loss & $210.89 \mathrm{~kW}$ & $141.63 \mathrm{~kW}$ & $102.66 \mathrm{~kW}$ \\
$\%$ reduction & - & $32.84 \%$ & $51.32 \%$ \\
Voltage dev. & $1.7720 \mathrm{pu}$ & $1.0991 \mathrm{pu}$ & 08180 \\
\% Improvement & - & $37.97 \%$ & $53.84 \%$ \\
LB index & 67.71 & 44.27 & 29.54 \\
$\%$ Improvement & - & $34.62 \%$ & $56.33 \%$ \\
Min. Voltage & $0.9108 \mathrm{pu}$ & $0.9413 \mathrm{pu}$ & 0.9624 \\
\hline
\end{tabular}

By varying the status of the switches during the reconfiguration has significantly improve the VP of the neighbouring buses. From Fig. 5, it can be seen that voltages of bus 10, 11 and 14 have greatly improved due to changes in $s 7, s 8$ and $s 15$ respectively. While the voltage magnitudes of bus $1-3$ remain constant because they are the feeder buses. It can also be observed that bus 12 has the lowest voltage magnitude, due to its remoteness from the substation and heavy load demand.

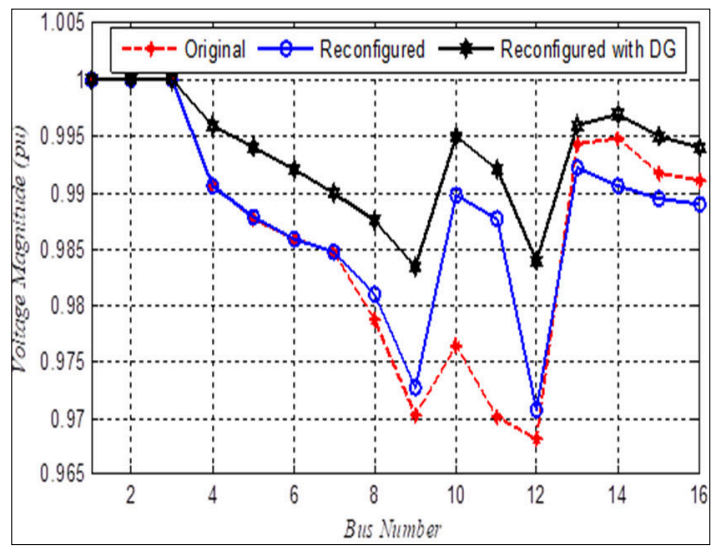

Fig . 5. Optimized 16-bus test system voltage profile.

\section{- 33-bus single-feeder DN}

In this case, the optimal DNR is obtained opening the switches $s 33-s 37$ while closing $s 7, s 11, s 14, s 28$ and $s 32$ as shown in Fig. 6. Similarly, the optimal locations of the RDG are found to be bus 14, 28 and 33 with sizes of $0.22 \mathrm{MW}$, $0.30 \mathrm{MW}$ and 0.24 respectively.

As result of this reconfiguration, the power loss is found to be reduced by $32.84 \%$ of the total inherent losses while the voltage deviation reduced by $37.97 \%$ of the initial deviation established to be $1.6610 \mathrm{pu}$, better than those obtained in [14]. With the RDG units placement, less power is transmitted along the branches implying less power loss and relieved feeder loading as summarised in Table 2.

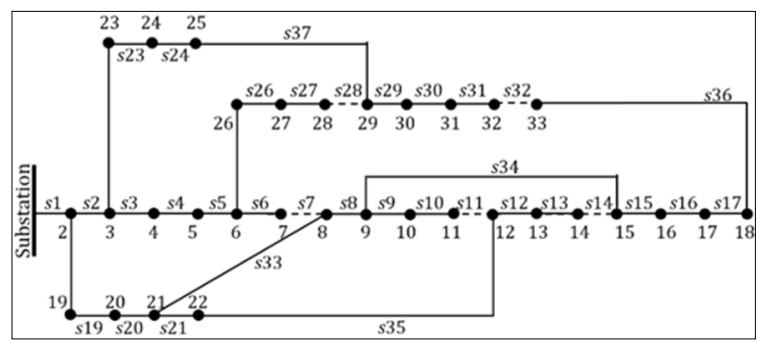

Fig. 6. Optimized configuration of 33-bus test system 
Table 3 69-Bus Test System Results

\begin{tabular}{lccc}
\hline \multicolumn{1}{c}{ Parameters } & Initial & Reconfigured & Reconfigured with RDG \\
\hline Tie switches & $69,70,71,72,73$ & $14,58,61,69,70$ & - \\
RDG location (Size) & - & - & Bus14 (0.44MW), bus35 (0.53MW), bus63 (0.61MW) \\
Power loss & $224.9804 \mathrm{~kW}$ & $151.07 \mathrm{~kW}$ & $120.88 \mathrm{~kW}$ \\
\% reduction & - & $32.84 \%$ & $46.27 \%$ \\
Voltage dev. & $0.2140 \mathrm{pu}$ & $0.1876 \mathrm{pu}$ & $0.1585 \mathrm{pu}$ \\
\% Improvement & - & $12.33 \%$ & $25.92 \%$ \\
LB index & 82.25 & 62.48 & 52.12 \\
\% Improvement & - & $24.03 \%$ & $36.65 \%$ \\
Min. Voltage & $0.9062 \mathrm{pu}$ & $0.9495 \mathrm{pu}$ & 0.9820 \\
\hline
\end{tabular}

By studying the system voltage profile, it can be seen that voltage magnitudes of bus 1,2 and 19 remain the same before and after reconfiguration and RDG placement. This is due to their proximity to the main substation. Conversely, the voltage magnitudes of bus $20-23$ have reduced following the reconfiguration. This is because of closing $s 33$ and $s 35$ thereby transferring loads on bus $8-18$ and 33 to the same feeder.

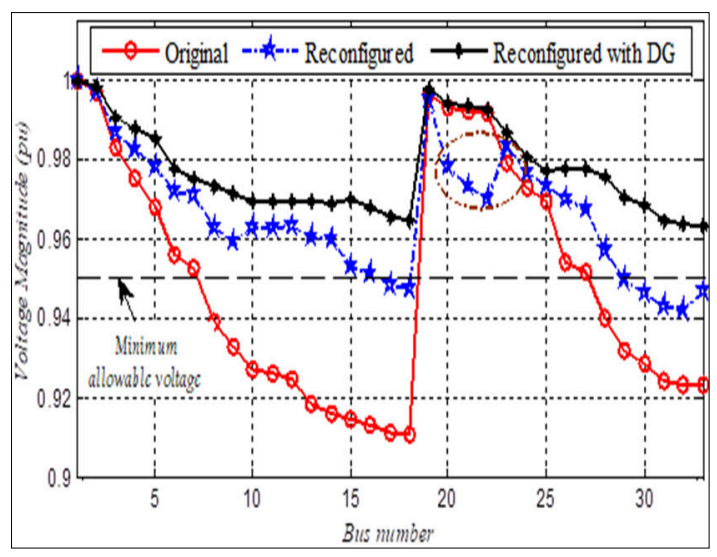

Fig. 7. Optimized 33-bus test system voltage profile.

\section{- $\quad$ 69-bus, Single f test system}

Despite the size of this system, the algorithm is capable of determining the optimal DNR and RDG locations and sizes concurrently for the minimization of the objective function subject to maintaining the radial network structure and the rest of the constraints. The optimal DNR is achieved by closing $s 71, s 72$ and $s 73$ and opening $s 14, s 58$ and $s 61$ while keeping $s 69$ and $s 70$ open as shown in Fig 8. Likewise, the optimal locations of the RDG are found to be bus 14, 35 and 63 , with sizes of $0.44 \mathrm{MW}, 0.53 \mathrm{MW}$ and $0.61 \mathrm{MW}$ respectively. The performance improvement obtained after the optimal DNR and simultaneous DNR and RDG placement are summarized in Table 3.

As result of this reconfiguration, the power loss, voltage deviation and LB are found to be reduced by 32.84 $\%, 12.33 \%$ and $24.03 \%$ of their respective inherent values. This improvement is much better than that obtained in [19, 20]. Additional significant improvement is observed in the objectives after the optimal placement of the RDGs.

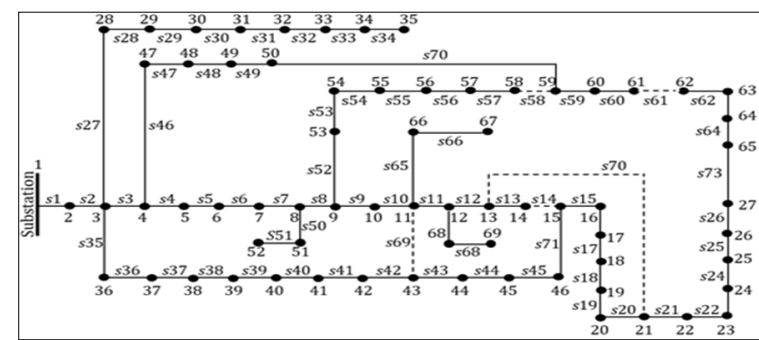

Fig. 8. Optimized configuration of 69 bus test system

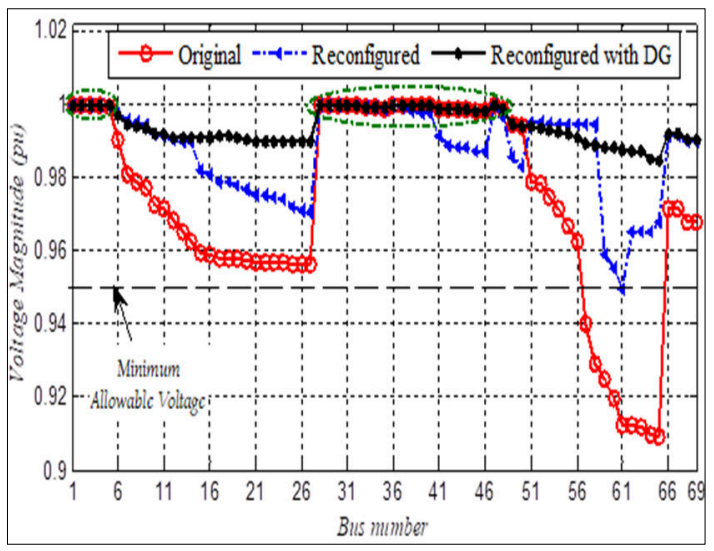

Fig. 9. Optimized 69-bus test system voltage profile.

By inspecting the voltage profile shown in Fig 9, voltage magnitudes of buses $41-46$ as has reduced significantly. This is caused as result of transferring some loads from middle feeder; loads at buses $15-27$ and buses $62-65$ to the feeder containing affect buses. For the same reason, voltages at bus 49 and 50 after reconfiguration is less than that before the configuration. Contrariwise, the voltage magnitudes of buses $1-6$ and buses $28-46$ remain unchanged after reconfiguring the system and placing the RDG units due to their proximity to the main feeder/ substation.

\section{CONCLUSIONS}

In this study, a hybrid of Particle Swarm Optimization (PSO) and real-coded Genetic Algorithm (GA) techniques is employed for DN reconfiguration and optimal allocation of three RDG units in primary DNs simultaneously. The 
objectives of the proposed technique are to minimize real power loss, improvement of voltage profile and feeder load balancing. For validation purpose, the proposed technique is implemented on 16-bus (3-feeder), single feeder 33-bus and 69-bus IEEE test DNs. It is implemented in MATLAB interfaced with MATPOWER simulation packages. From the simulation result, the technique is capable of reconfiguring the networks effectively, determined the optimal locations and sizes of three RDG units thereby minimizing the objectives. With the optimal RDG placement and reconfiguration, the radial structure is preserved in all the scenarios, no loop is formed. Despite the size of 69 bus system, the result obtained is quite appreciable. It is observed the reduction in PL, improvement in VP and LB are found to be $32.84 \%, 12.33 \%$ and $24.03 \%$ with respect to inherent values in the biggest system when the system is reconfigured only. With the optimized RDGs placed in the reconfigured systems, a further reductions of $46.27 \%, 25.92 \%$ and $36.65 \%$ are observed respectively.

It is envisioned as future work to carry out the optimal DNR and RDG allocation dynamically/online while maximizing the penetration level of the RDG units.

\section{AUTHOR INFORMATION}

\section{Corresponding Author}

*Email: abkunya@abu.edu.ng

\section{ORCID}

A.B. Kunya : 0000-0001-5558-358X

A.U. Lawan : 0000-0002-0789-3679

\section{REFERENCES}

[1] Gomez-Gonzalez M, López A, Jurado F. Optimization of distributed generation systems using a new discrete PSO and OPF. Electric Power Systems Research. 2012;84(1):174-80.

[2] Georgilakis P.S, Hatziargyriou N.D. Optimal Distributed Generation Placement in Power Distribution Networks: Models, Methods, and Future Research. IEEE Transactions on Power Systems. 2013;28(3):3420-8.

[3] de Oliveira,L.W, Seta F.d.S, de Oliveira E.J. Optimal reconfiguration of distribution systems with representation of uncertainties through interval analysis. International Journal of Electrical Power \& Energy Systems. 2016;83:382-91.

[4] Fathabadi H. Power distribution network reconfiguration for power loss minimization using novel dynamic fuzzy c-means (dFCM) clustering based ANN approach. International Journal of Electrical Power \& Energy Systems. 2016;78:96-107.

[5] Borozan V, Rajicic D, Ackovski R. Minimum loss reconfiguration of unbalanced distribution networks. IEEE Transactions on Power Delivery. 1997;12 (1):435-42.

[6] Baran M.E, Wu F.F. Network reconfiguration in distribution systems for loss reduction and load balancing. IEEE Transactions on Power Delivery. 1989;4(2):1401-7.
[7] Ghasemi S. Balanced and unbalanced distribution networks reconfiguration considering reliability indices. Ain Shams Engineering Journal. 2018;9 (4):1567-79.

[8] Raju G.K.V, Bijwe P.R. Efficient reconfiguration of balanced and unbalanced distribution systems for loss minimisation. IET Generation, Transmission \& Distribution. 2008;2(1):7-12.

[9] Nguyen T.T, Truong A.V. Distribution network reconfiguration for power loss minimization and voltage profile improvement using cuckoo search algorithm. International Journal of Electrical Power \& Energy Systems. 2015;68:233-42.

[10] Shehu G. Distribution Network Reconfiguration for Loss Reduction and Voltage Profile Improvement using B-PSO2016.

[11] Ch Y, Goswami S.K, Chatterjee D. Effect of network reconfiguration on power quality of distribution system. International Journal of Electrical Power \& Energy Systems. 2016;83:87-95.

[12] Zhai H.F, Yang M, Chen B, Kang N. Dynamic reconfiguration of three-phase unbalanced distribution networks. International Journal of Electrical Power \& Energy Systems. 2018;99:1-10.

[13] Kianmehr E Nikkhah S, Rabiee A. Multi-objective stochastic model for joint optimal allocation of DG units and network reconfiguration from DG owner's and DisCo's perspectives. Renewable Energy. 2019; 132:471-85.

[14] Capitanescu F, Ochoa L.F, Margossian H, Hatziargyriou ND. Assessing the Potential of Network Reconfiguration to Improve Distributed Generation Hosting Capacity in Active Distribution Systems. IEEE Transactions on Power Systems. 2015;30 (1):346-56.

[15] Singh S.P, Raju G.S, Rao G.K, Afsari M. A heuristic method for feeder reconfiguration and service restoration in distribution networks. International Journal of Electrical Power \& Energy Systems. 2009;31(7):309-14.

[16] Qin Z, Shirmohammadi D, Liu W.E. Distribution feeder reconfiguration for service restoration and load balancing. IEEE Transactions on Power Systems. 1997;12(2):724-9.

[17] $\mathrm{Zu} \mathrm{G,} \mathrm{Xiao} \mathrm{J,} \mathrm{Sun} \mathrm{K.} \mathrm{Distribution} \mathrm{network}$ reconfiguration comprehensively considering $\mathrm{N}-1$ security and network loss. IET Generation, Transmission \& Distribution. 2018;12(8):1721-8.

[18] Nguyen T.T, Nguyen T,T, Truong A.V, Nguyen Q.T, Phung T.A. Multi-objective electric distribution network reconfiguration solution using runner-root algorithm. Applied Soft Computing. 2017;52:93-108.

[19] Jabr R.A, Singh R, Pal B.C. Minimum Loss Network Reconfiguration Using Mixed-Integer Convex Programming. IEEE Transactions on Power Systems. 2012;27(2):1106-15.

[20] Mahboubi-Moghaddam E, Narimani M.R, Khooban M.H, Azizivahed A, Javid sharifi M. Multi-Objective Distribution feeder reconfiguration to improve transient stability, and minimize power loss and 
operation cost using an enhanced evolutionary algorithm at the presence of distributed generations. International Journal of Electrical Power \& Energy Systems. 2016;76:35-43.

[21] Merlin A, Back H. Search for a minimal loss operating spanning tree configuration in an urban power distribution system. In Proceeding 5th power syst comput conf (PSCC). Cambridge. UK. 1975;1-18

[22] Lee C, Liu C, Mehrotra S, Bie Z. Robust Distribution Network Reconfiguration. IEEE Transactions on Smart Grid. 2015;6(2):836-42.

[23] Kavousi-Fard A, Abbasi A, Rostami M-A, Khosravi A. Optimal distribution feeder reconfiguration for increasing the penetration of plug-in electric vehicles and minimizing network costs. Energy. 2015;93: 1693-703.

[24] Niknam T. An efficient hybrid evolutionary algorithm based on PSO and HBMO algorithms for multi-objective Distribution Feeder Reconfiguration. Energy Conversion and Management. 2009;50 (8):2074-82.

[25] Moshtagh J, Ghasemi S. Optimal Distribution System Reconfiguration Using Non-dominated Sorting Genetic Algorithm (NSGA-II). Journal of Operation and Automation in Power Engineering. 2007;1(1): 12-21.

[26] Rao P, Sivanagaraju S. Radial Distribution Network Reconfiguration for Loss Reduction and Load Balancing using Plant Growth Simulation Algorithm. International Journal on Electrical Engineering and Informatics. 2010;2.

[27] Saffar A, Hooshmand R, Khodabakhshian A. A new fuzzy optimal reconfiguration of distribution systems for loss reduction and load balancing using ant colony search-based algorithm. Applied Soft Computing. 2011;11(5):4021-8.

[28] Niknam T. An efficient multi-objective HBMO algorithm for distribution feeder reconfiguration. Expert Systems with Applications. 2011;38(3): 2878-87.

[29] Ahuja A, Das S, Pahwa A. An AIS-ACO Hybrid Approach for Multi-Objective Distribution System Reconfiguration. IEEE Transactions on Power Systems. 2007;22(3):1101-11.

[30] Alonso F.R, Oliveira D.Q Souza A.C.Z.d. Artificial Immune Systems Optimization Approach for Multiobjective Distribution System Reconfiguration.
IEEE Transactions on Power Systems. 2015;30 (2):840-7.

[31] Andervazh M, Olamaei J, Haghifam M. Adaptive multi-objective distribution network reconfiguration using multi-objective discrete particles swarm optimisation algorithm and graph theory. IET Generation, Transmission \& Distribution. 2013;7 (12):1367-82.

[32] Ud Din F, Ahmad A, Ullah H, Khan A, Umer T, Wan S. Efficient sizing and placement of distributed generators in cyber-physical power systems. Journal of Systems Architecture. 2019;97:197-207.

[33] Hadidian-Moghaddam M.J, Arabi-Nowdeh S, Bigdeli M, Azizian D. A multi-objective optimal sizing and siting of distributed generation using ant lion optimization technique. Ain Shams Engineering Journal. 2018;9(4):2101-9.

[34] Rama P.D, Jayabarathi T. Optimal placement and sizing of multiple distributed generating units in distribution networks by invasive weed optimization algorithm. Ain Shams Engineering Journal. 2016;7 (2):683-94.

[35] Nguyen T.T, Truong A.V, Phung T.A. A novel method based on adaptive cuckoo search for optimal network reconfiguration and distributed generation allocation in distribution network. International Journal of Electrical Power \& Energy Systems. 2016;78:801-15.

[36] Tolabi H.B, Ali M.H, Rizwan M. Simultaneous Reconfiguration, Optimal Placement of DSTATCOM, and Photovoltaic Array in a Distribution System Based on Fuzzy-ACO Approach. IEEE Transactions on Sustainable Energy. 2015;6 (1):210-8.

[37] Ali A.F, Tawhid M.A. A hybrid particle swarm optimization and genetic algorithm with population partitioning for large scale optimization problems. Ain Shams Engineering Journal. 2017;8(2):191-206.

[38] Le A.D.T, Kashem M.A, Negnevitsky M, Ledwich G, editors. Maximising Voltage Support in Distribution Systems by Distributed Generation. TENCON 2005 - 2005 IEEE Region 10 Conference; 2005 21-24 Nov. 2005.

This article is licensed under a Creative Commons Attriution 4.0 International License. 